\title{
Study on altitude control of Insect-Like Flapping-Wing Micro Aerial Vehicle
}

\author{
Zheng Zhang, Wei-pingZhang*, Xi-junKe, YangZou, NanHu, FanWu \\ (National Key Laboratory of Science and Technology on Micro/Nano Fabrication Laboratory, \\ Laboratory for Thin Film and Micro fabrication of the Ministry of Education, Department of \\ Micro-Nano Electronics, School of electronic information and electrical engineering, Shanghai Jiao \\ Tong University, Shanghai 200240, China)
}

Keywords:flapping-wing micro aerial vehicle,altitude control,system identification.

\begin{abstract}
Altitude control of insect-like Flapping-Wing Micro Aerial Vehicles is an intrinsically multidisciplinary problem, in which several engineering aspects must be considered simultaneously, including aerodynamics, sensing, signal generation, micro-fabrication and control theory. Because of complexity and uncertainty of aerodynamic modeling, we used the method of combining theoretical analysis and system identification experiment. In the first stage, according to theoretical analysis of the lift force model, amplitude modulation and frequency modulation is the key method to modulate lift force. Then obtained static system dynamics model by system identification experiment, and got its resonant frequency is $129 \mathrm{~Hz}$ from the bode diagram. The design ofthe controller relies on the modified identified model which is treat an excitation signal as a subsystem of the vehicle, avoiding measurement of the piezoelectric actuator displacement in flight. This work is a key promotion and also is of great significance to realize auto-controlled flight of FWMAV.
\end{abstract}

\section{Introduction}

Research on the flight began with mankind's awe and envy of flying creatures. Further understanding of aerodynamics and development of microfabrication technology has enabled Insect-Like Flapping-Wing Micro Aerial Vehicle (FWMAV).

In [1], the feasibility of flying robotic insects was empirically demonstrated. The lift-off of a milligram scale fly shows that insect-like flapping-wing micro aerial vehicle can generate lift forces sufficiently large to overcome gravity. However, the control challenges that need to be solved in order to achieve total autonomy of the vehicle are both attractive and formidable. Many fly insects are agile and maneuverable, it is natural to look to bio-inspired both morphology control strategies, researchers have attempted to illuminate the control principle of flying insects. In [2], researchers primarily formulated the aerodynamic model of the micro flapping wing aerial vehicle, then based on the dynamic model, put forward an uncoupled control matrix and lastly attested the control algorithm with simulation. But this method estimates the interference and damping of the actual flight is insufficient and inaccurate, and also limited to their own flapping wing micro aerial vehicle cannot overcome gravity, the algorithm isn't verified by experiment.

In this paper, we introduce a methodology for designing controllers capable of ascending in vertical flight and hovering. The problem is settled in two steps. In the first step, theoretical analysis the lift force model of FWMAV is illuminated which translate altitude control into amplitude modulation and frequency modulation of the exciting signal. In the second step, system identification of the actuator-transmission-wing system is performed which giving bandwidth and natural frequency of the system. The information gathered through the experiment is used for designing controller.

\section{Structure and aerodynamic analysis of FWMAV}

The insect-like FWMAV consists of four main components (Fig.1), each analogous to a biological counterpart: airframe (exoskeleton), actuator (muscles), transmission (thorax), airfoils (wings). The actuator, a clamped-free piezoelectric cantilever beam, provides an oscillatory mechanical input to a 
symmetric fourbar transmission, which converts linear motion of the tip motion of the actuator to flapping stroke of the wings.

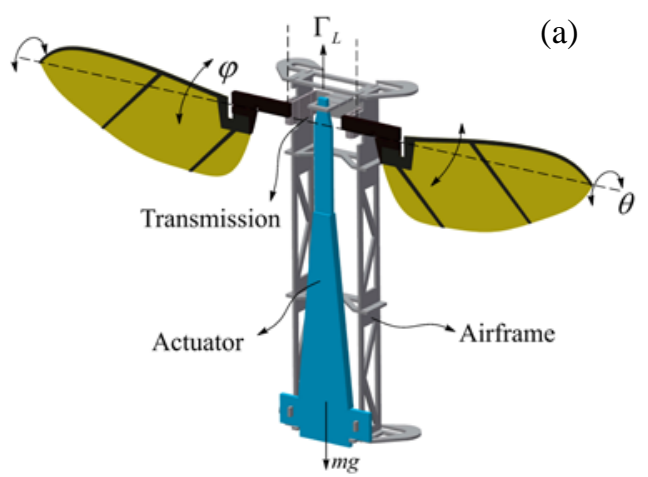

(b)

Fig. 1 (a)Structure and simple body coordinate system of FWMAV (b) Illustration of wing parameters

The specific objective of the research is present a methodto design altitude controlstrategy for the FWMAV in figure 1(a) constrained to movealong a vertical axis. The analysis begins with the free bodycoordinate of the FWMAV shown in figure 1(a). A first thing tonote is that from figure 1(a), the equation of motion along thevertical axis is simply

$$
\gamma_{L}(\tau)-m g=m x^{\prime \prime}(\tau)
$$

Where $m$ is mass of the FWMAV, $g$ is the gravitationalacceleration and $\gamma_{L}(\tau)$ is the instantaneous lift force generatedby the flapping wings. In some cases, an additional dissipativebody drag term $\kappa_{d} x^{\prime}(\tau)$ could be added to the right side of (1), where $\kappa_{d}$ is a constant to be confirmed experimentally. Notethat (1) is unstable because itsinput-output representation has two poles at 0 . As discussed in [3-4], there are several different points of view. Generally, $\gamma_{L}(\tau)$ is formulated as a nonlinear function of frequency and amplitude of the flapping stroke $\phi$. When the actuator signal is sinusoidal, $\gamma_{L}(\tau)$ will typically oscillatearound some non-zero mean force, crossing zero periodically. Therefore, ascent occurs when the average lift force $\Gamma_{L}$ is largerthan $m g$.

As described in [5], the mean total force $\Phi_{T}$, generated by passive rotation of a symmetrical wing pair throughout the flapping stroke is estimated as

$$
\Phi_{T}=\int_{0}^{L} \rho \bar{C}_{\Phi} \overline{v_{r}^{2}}(\xi) c(\xi) \mathrm{d} \xi
$$

which is a standard quasi-steady blade-element formulation offlight force, where $\rho$ is thedensity of the air, $\bar{C}_{\Phi}$ is the mean aerodynamic forcecoefficient of the wing throughout the stroke, $\overline{v_{r}^{2}}(\xi)$ is themean square relative velocity of each wing section, $c(\xi)$ is thechord length of the wing at a distance $\xi$ from the base, and $L$ is the total wing length (Illustration of wing parameters see Fig.1(b)). Note that assuming a horizontalstroke plane, for a sinusoidal stroke $\varphi(\tau)=\varphi_{0} \sin \left(2 \pi f_{r} \tau\right)$, the relative velocity of the wing plane can be estimated as

$$
v_{r}(\tau, \xi)=\xi \varphi^{\prime}(\tau)=2 \pi f_{r} \xi \varphi_{0} \cos \left(2 \pi f_{r} \tau\right)
$$

which implies that the mean square relative velocity of eachwing plane can be roughly estimated as

$$
\overline{v_{r}^{2}}(\xi)=4 \pi^{2} f_{r}^{2} \xi^{2} \varphi_{0}^{2} \frac{1}{T_{r}} \int_{0}^{T_{r}} \cos ^{2}\left(2 \pi f_{r} \tau\right) \mathrm{d} \tau
$$

with $T_{r}=f_{r}^{-1}$. Thus, it immediately follows that

$$
\overline{v_{r}^{2}}(\xi)=2 \pi^{2} f_{r}^{2} \xi^{2} \varphi_{0}^{2}
$$

As discussed above, regardless of the size and shape of the wing, $\Phi_{T}$ directly depends on $f_{r}^{2}$ and $\varphi_{0}^{2}$. This indicates that we can modulate the average lift force bychanging the stroke amplitude $\phi_{0}$, or 
by changing the strokefrequency $f_{r}$. In the problem considered in this paper, (2) is not practical to design a general control strategy, because itdepends on the morphology of insects. However, (5) is a guide which we can inspire control strategies. Transmission of the FWMAV can be approximated by a constant $\kappa$, it implies that maps from actuator output $y(t)$ to flapping angle $\varphi(t)$ can be expressed as $\varphi(t)=\kappa y(t)$. Thus, changing the frequency and/or amplitude of $y(t)$ can be modulate $\Phi_{T}$.

\section{System identification and LTI modelling of FWMAV}

\subsection{Problem formulation and experiment}

According to structure of FWMAV shown in figure 1(a), we can extract two relevant mapping relation. One is the dynamic mapping from the input voltage to the actuatordisplacement, labeledas $P$, whose idealized block diagram is shown in figure 2.Here, $y(t)$ is the input, $y(t)$ is the measured displacement of actuator and $v(t)$ is output disturbance, representingall the disturbances affecting the system, including vibrationsgenerated by the aerodynamic forces produced by the wingflapping. The other is mapping from actuator displacement $y(t)$ to steady-state average lift force $\Gamma_{L}$, labeled as $\Psi(A, f)=\Gamma_{L}$, where $A$ and $f$ is the amplitude and frequency of $y(t)=A \sin (2 \pi f t)$ respectively. Actually, the second mapping exactlyreflect the lift force model discussed before. The transfer function $P$ is significant because it represent the bandwidth and natural frequency of the system. Also it can be used to design controller. The mapping $\Psi$ is relevant because it helps us to ascertain suitable input frequencies and amplitudes.

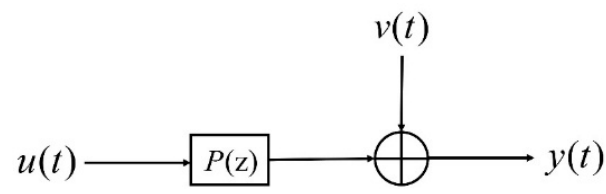

Fig. 2Idealized system dynamics of FWMAV.

Estimation of $P(\mathrm{z})$ is obtained by system identification experiment. Illustration of experimental setup is depicted in figure 3(a). Here, the FWMAV is rigidly fixed on the supporting beam, simultaneously, a noncontact charge-coupled laser displacement sensor is used to measure the tip displacement of the piezoelectric actuator. In order to reduce disturbance from micro-vibration of environment, whole system is installed on the shockproof platform. Figure 3(b) is a picture of the experimental setup for system identification. $u(t)$ is 10000 samples generated using white-noise signal at a sampling-and-hold rate of $10 \mathrm{KHz} . y(t)$ is measured by short-rangelaser displacement sensor LK-G30 fabricated by Keyence(capacity: 10mm, precision: 0.05um).

(a)
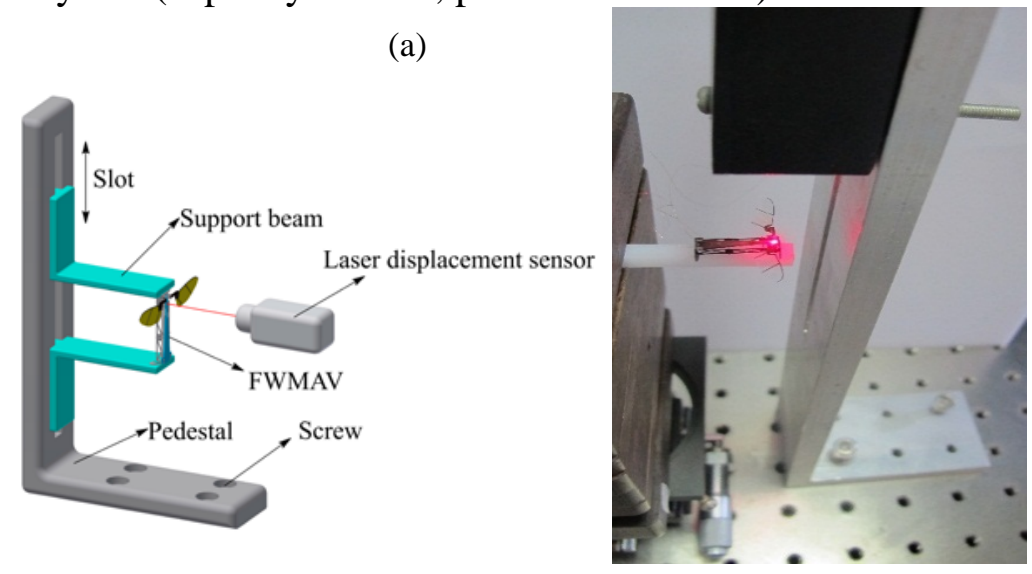

(b)

\subsection{Results and frequency response}

Using MATLAB system identification toolbox and $n 4$ sid algorithm process the data which captured by experiment. The resulting identified LTI system dynamics $\hat{P}(\mathrm{z})$ are given by 
With the matrix

$$
\begin{array}{r}
x_{P}(k+1)=A_{P} x_{P}(k)+B_{P} u(k) \\
y(k)=C_{P} x_{P}(k)+D_{P} u(k)
\end{array}
$$

$$
\begin{aligned}
A_{P} & =\left[\begin{array}{llcl}
0.99190 & 0.10055 & 0 & 0.00849 \\
0.06821 & 0.93644 & 0.00004 & 0.02358 \\
0.00001 & 0.00001 & 1.00001 & 0.00003 \\
0.00038 & 0.04305 & 0.00001 & 0.99786
\end{array}\right] \quad B_{P}=\left[\begin{array}{c}
0.00021 \\
0.00109 \\
0 \\
-0.00015
\end{array}\right] \\
C_{P} & =\left[\begin{array}{llll}
-0.20813 & 0.06303 & 0.00001 & 0.07886
\end{array}\right] \\
D_{P} & =0
\end{aligned}
$$

Fig.4is Bode diagram of identified dynamic model $\hat{P}(\mathrm{z})$, we can obtain the natural frequency of $\hat{P}(\mathrm{z})$ is $129 \mathrm{~Hz}$. Notice that since the system identification experiment is performed withthe actuator mounted to the airframe and connected to the transmission mechanism, the frequency response in Fig.4 does notcapture the dynamics of the actuator, but the coupled dynamicsof the actuator-transmission-wing-airframe system. Furthermore, on account of difference micromachining process and assembling, dynamic model in this paper are used to propose control strategies, but they don't represent the typical dynamics of FWMAV.

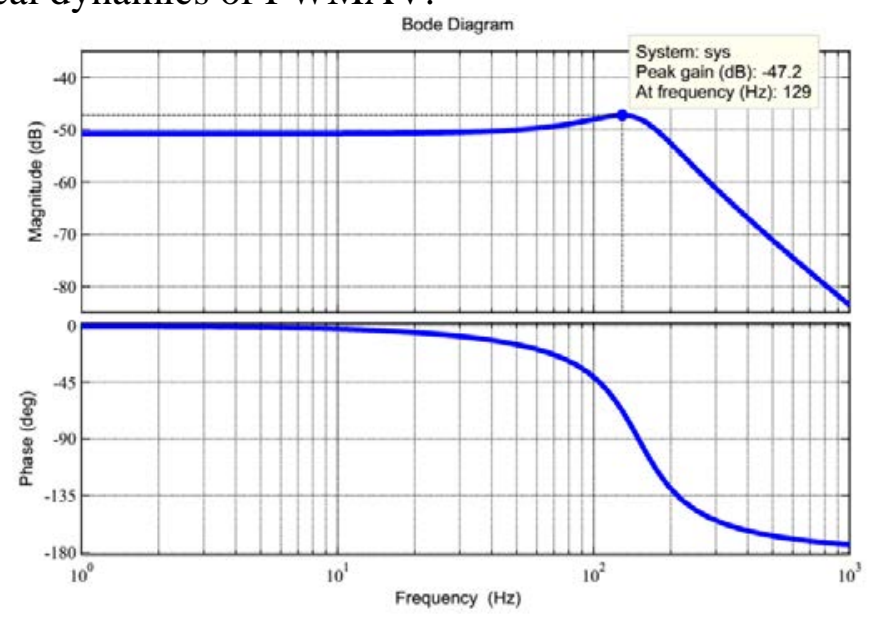

Fig. 4Bode diagram of identified system dynamics $\hat{P}$

\section{Control Strategies}

In the previous section, we captured dynamics model from input voltage to actuator distal displacement by system identification experiment, and obtained the natural frequency of the model. In order to implement a feedback control loop around $P(\mathrm{z})$, a measurement of the actuator distal displacement is required. However, in flight stage, displacement sensors for real-time and accuracy measuringthe actuator output is infeasible. With the available technology, we capable of real-time measurement of FWMAV vertical height. Thus, real-time height can be the control variables also the feedback variables. Inspired not only by nature, but also for practicalreasons, roboticists have commonly designed flapping-wingmechanisms to be excited by sinusoidal signals. Here, weadopt the same design choicearbitrarily, acknowledging that other shapesfor exciting signals are conceivable.

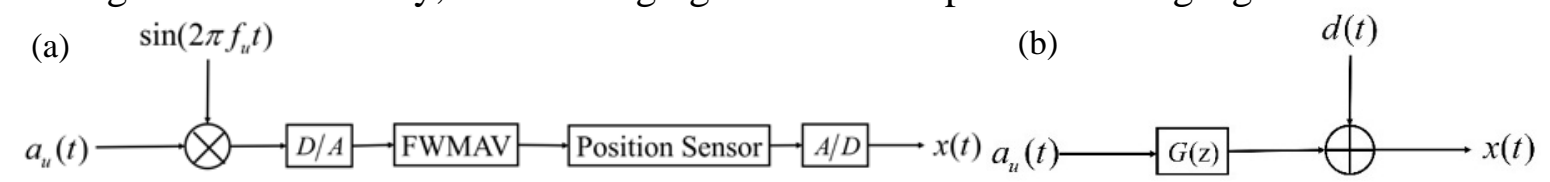

Fig. 5 (a)Open-loop block diagram of the system used in altitude control, (b) Idealized block diagram

As mentioned above, an open-loop altitude control system was designed, depicted in Fig. 5(a) which can be idealized by block diagram in Fig.5 (b). The idea is to treat $\sin \left(2 \pi f_{u} t\right)$ as part of the 
system, consider $a_{u}(t)$ to be the control signal and $f_{u}$ is fixed frequency. This method fixed frequency-amplitude modulationwhich is mentioned in section 2. Here, $D / A$ representsthe digital-to-analog signal conversion and the amplificationfrom $a_{u}(t)$ to the analog range $[0,300] \mathrm{V}$, $A$ / $D$ is opposite, represents the analog-to- digital signal conversion, $x(t)$ is altitude height measured by long-range laser displacement sensor, $G(\mathrm{z})$ is discrete-time open-loop plant of the idealized system dynamics, $d(t)$ represents all disturbancesaffecting the system including sensor noise. Then, we can implement a feedback controller around $G(\mathrm{z})$ with Simulink model and PID Tuner toolbox, block diagram of the design is shown in figure 6.

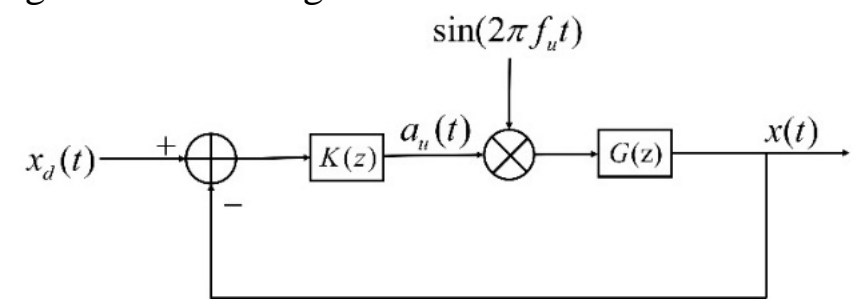

Fig. 6Block diagram of feedback controller design.

\section{Summary}

In this article, we presented an investigation on the issue of altitude control problem in one degree of freedom. Firstly, average lift modelwas established based on blade element method, we draw a conclusion that changing the input signal amplitude or frequency can modulate lift force. Then, we built the coupled dynamics of the actuator-transmission-wing-airframe system by system identification experiment and captured the natural frequency of the system $129 \mathrm{~Hz}$. In order to implement a feedback control loop, we modified the map $P(\mathrm{z})$ from input voltage to actuator displacement to the map $G(\mathrm{z})$ from amplitude of excitation signal to FWMAV real-time altitude height because measurement of the distal displacement is infeasible while the FWMAV flying. In the end, we introduced a method of designing controller using classical techniques.

As mentioned in the beginning of the article, the control problem demanding prompt solution. Theresults presented here suggest that the approach introduced inthis investigation is appropriate in the pursuit of completelyautonomous flying FWMAV.

\section{Acknowledgements:}

This research was financially supported by the Supporting Foundation of the Ministry of Education (62501040303), the Pre-research Fund ( 9140 A 2602031 3JW 03 371, 9140 A 26020 414J W 03412 ), and the New Century Excellent Talents Support Program from the Ministry of Education of China (NCET-10-0583).

*Corresponding author: zhangwp@sjtu.edu.cn

\section{References}

[1] Wood R J. The First Takeoff of a Biologically Inspired At-Scale Robotic Insect[J]. IEEE Transactions on Robotics, 2008, 24(2):341-347.

[2] Doman D B, Oppenheimer M W, Sigthorsson D O. Wingbeat Shape Modulation for Flapping-Wing Micro-Air-Vehicle Control During Hover[J]. Journal of Guidance Control \& Dynamics, 2012, 33(3):724-739.

[3] Deng X, Schenato L, Wu W C, et al. Flapping flight for biomimetic robotic insects: part I-system modeling[J]. Robotics IEEE Transactions on, 2006, 22(4):776 - 788. 
[4] Lehmann F O. The control of wing kinematics and flight forces in fruit flies (Drosophila spp.).[J]. Journal of Experimental Biology, 1998, 201(3):385-401.

[5] Ellington C P. The Aerodynamics of Hovering Insect Flight[J]. II Morphological, 1984. 